\title{
EFECTO DE LA PREDNISONA Y SU RELACIÓN CON LA LONGITUD DEL MOVIMIENTO DENTAL EN RATAS (Rattus norvegicus)
}

\author{
Effect of prednisone and relation on length of tooth movement in rats (Rattus norvegicus)
}

\author{
Eliberto Ruiz ${ }^{1}$, Jorge L. Arroyo ${ }^{2}$, Martin Condorhuamán ${ }^{3}$ \\ ${ }^{1}$ Facultad de Farmacia y Bioquímica. ${ }^{2}$ Facultad de Medicina Humana. ${ }^{3}$ Instituto de Investigación en Ciencias Farmacéuticas \\ y Recursos Naturales "Juan de Dios Guevara", Facultad de Farmacia y Bioquímica \\ Universidad Nacional Mayor de San Marcos
}

\section{RESUMEN}

El presente estudio de tipo experimental, tuvo como objetivo evaluar el efecto de la prednisona sobre la relación de la longitud del movimiento dental en ratas (Rattus norvegicus). La evaluación de dicha relación se realizó en 20 ratas machos Wistar de 12 semanas de edad, con un peso de $225 \pm 25 \mathrm{~g}$, a las cuales se les adapto un aparato disyuntor ortodóntico en sus incisivos centrales superiores, luego fueron divididos de manera aleatoria en dos grupos: control (suero fisiológico a 2 mL/ $\mathrm{kg}$ ) y experimental (prednisona $20 \mathrm{mg} / \mathrm{kg}$ vía intraperitoneal). El tratamiento se inicio cinco días antes de la colocación del aparato y luego al primer, tercer y quinto día. Con un calibrador, se midió la distancia interincisiva al terminar de colocar el aparato, así como al primer, tercer, quinto y séptimo día. En el 1er día de evaluación, el grupo control y el grupo experimental tuvieron una distancia interincisiva de 1,13 $\pm 0,18 \mathrm{~mm}$ y 0,24 $\pm 0,09 \mathrm{~mm}$ y al $7 \mathrm{mo}$ día fue de 1,76 \pm 0,14 $\mathrm{mm}$ y 1,56 \pm 0,20 $\mathrm{mm}$, respectivamente, siendo estas diferencias significativas en el primer y séptimo día ( $<<0,05)$. Se concluye que la prednisona reduce significativamente el movimiento dental en ratas.

Palabras clave: Prednisona, movimiento dental, disyuntor ortodóntico, distancia interincisiva, Rattus norvegicus.

\section{SUMMARY}

This experimental study was to evaluate the effect of prednisone on the ratio of the length of tooth movement in rats (Rattus norvegicus). The evaluation of this relationship was performed in 20 male Wistar rats 12 weeks of age, weighing 225 $\pm 25 \mathrm{~g}$, to which adapted a orthodontic breaker in their upper central incisors, were then randomly divided into two groups: control (physiological saline to $2 \mathrm{~mL} / \mathrm{kg}$ ) and experimental (prednisone $20 \mathrm{mg} / \mathrm{kg}$ ip), the treatment began five days before placement of the device and then to the first, third and fifth day. With a gauge, was measured the interincisisor distance to finish placing the device, as so as to the first, third, fifth and seventh day. On the ist day of testing, the control group and the experimental group had a interincisiva distance $1,13 \pm 0,18 \mathrm{~mm}$ and $0,24 \pm 0,09 \mathrm{~mm}$ and the 7 th day was $1,76 \pm 0,14 \mathrm{~mm}$ and $1,56 \pm 0,20 \mathrm{~mm}$, respectively, with significant differences in the first and seventh days $(p<0,05)$. It's conclude that prednisone significantly reduces tooth movement in rats.

Keywords: Prednisone, tooth movement, orthodontic breaker, interincisor distance, Rattus norvegicus.

\section{INTRODUCCIÓN}

a modificación del movimiento dental es un problema con el que frecuentemente se enfrentan los ortodoncistas. La ortodoncia es una especialidad de la odontología que busca corregir la mala posición dental y ósea en pacientes que padecen dichas alteraciones ${ }^{(1)}$. En numerosas ocasiones los ortodoncistas atienden en la práctica clínica a personas que están recibiendo tratamiento farmacológico, que puede incluir glucocorticoides, por alguna enfermedad sistémica. Una pregunta importante a contestar, en estos casos, sería ¿en qué medida afecta la medicación con glucocorticoides al movimiento dental?, ya que la respuesta sería muy útil para predecir el tiempo que durará el tratamiento ortodóntico en estos pacientes debido a que, al parecer, su evolución es distinta a quienes no son tratados con dichos medicamentos.

Los glucocorticoides están involucrados en la fisiología ósea, asimismo, los osteoblastos y los osteoclastos pueden expresar receptores para estos compuestos, lo cual es influenciado por factores proinflamatorios, como las interleuquinas 6 y 11.

La prednisona es un glucocorticoide de acción intermedia muyútil dentro del tratamiento de patologías crónicas, pudiendo estar indicado en pacientes bajo tratamiento de insuficiencia adrenocortical aguda o primaria crónica, síndrome adrenogenital, anemia hemolítica adquirida, anemia hipoplásica congénita, trombocitopenia secundaria en adultos, enfermedades alérgicas, del colágeno, reumáticas, respiratorias, 


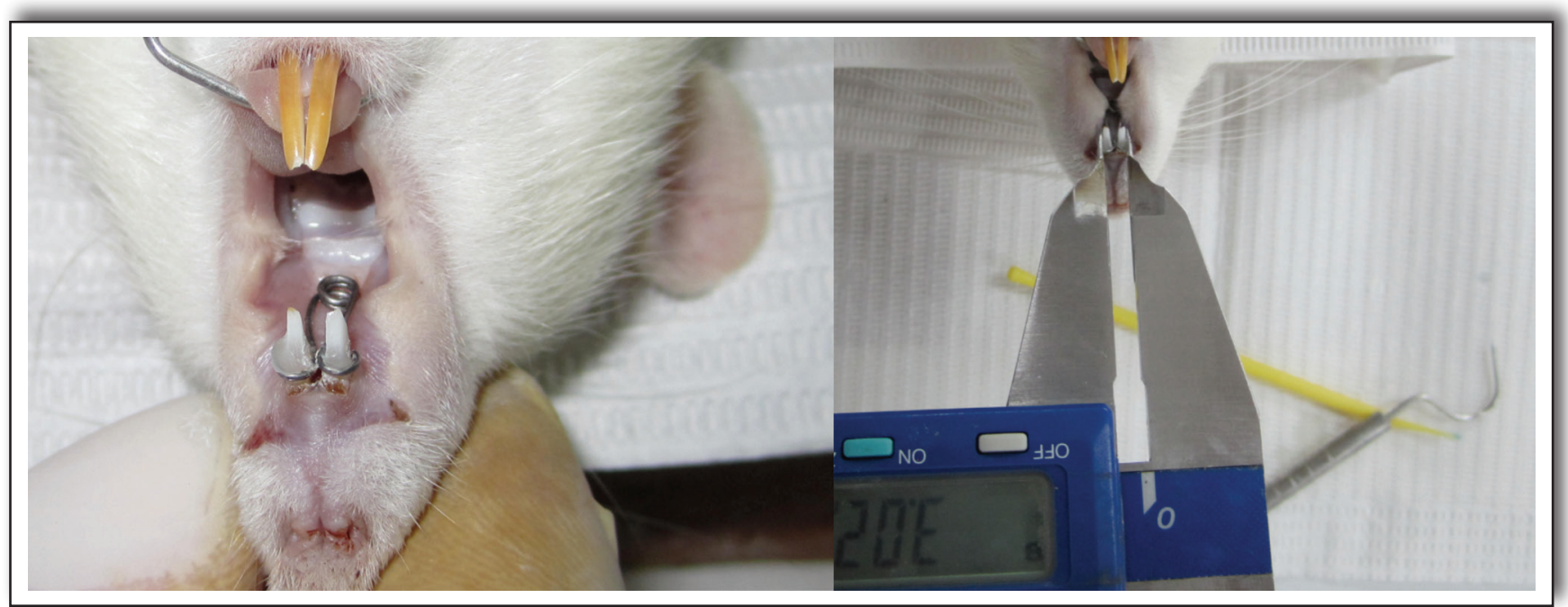

Figura 1. Visualización del disyuntor ortodóntico y medición de la longitud interdental.

gastrointestinales, neoplásicas y estados edematosos ${ }^{(2)}$.

El objetivo del presente estudio fue evaluar el efecto de la prednisona sobre la relación de la longitud del movimiento dental en ratas (Rattus norvegicus) y así tener una mejor proyección de los resultados en el tratamiento ortodóntico de pacientes que reciben dicho medicamento.

\section{MATERIAL Y MÉTODOS}

La presente investigación es de tipo experimental, prospectiva y longitudinal. Se realizó en el bioterio y laboratorio de farmacología de la Facultad de Medicina de la Universidad Nacional Mayor de San Marcos.

Se utilizaron 20 ratas macho (Rattus norvegicus) Wistar de 12 semanas de edad, con un peso de $225 \pm 25$ g adquiridas del Instituto Nacional de Salud. Se les mantuvo en el lugar de trabajo por siete días previos a la experimentación. El ambiente estuvo a una temperatura entre $19^{\circ}$ a $22^{\circ} \mathrm{C}$, con una humedad de $60 \%$ a $70 \%$ y con periodos de luz/oscuridad de 12 horas, proporcionándoles alimento y agua.

\section{Determinación del movimiento dental}

Los animales fueron divididos aleatoriamente en dos grupos de diez ratas cada uno, considerándose un grupo control que recibió suero fisiológico a $2 \mathrm{~mL} / \mathrm{kg}$ y un grupo experimental que recibió prednisona a $20 \mathrm{mg} /$ $\mathrm{kg}$. Todos los animales fueron anestesiados utilizando pentobarbital sódico a $30 \mathrm{mg} / \mathrm{kg}$ porvía intraperitoneal. Sedeterminóla distancia entrelosángulos incisodistales delosincisivos maxilaresutilizandoun calibradordigital de $0,01 \mathrm{~mm}$ de precisión. Los incisivos maxilares de todas las ratas fueron desgastados superficialmente en la cara mesial, con una fresa diamantada de fisura de o, $5 \mathrm{~mm}$ de diámetro para poder colocar el disyuntor ortodóntico. El dispositivo ortodóntico utilizado fue un doblez de tres vueltas, de dos mm de diámetro, con brazos de diez $\mathrm{mm}$ de longitud, de alambre o,016 de aleación beta-titanio. Con un dinamómetro se reguló una tensión de $35 \mathrm{~g}$ en el dispositivo

Tabla 1. Distancia interincisiva $(\mathrm{mm})$ según tratamiento y por días de evaluación.*

\begin{tabular}{cccc}
\hline & $\begin{array}{c}\text { Control } \\
\mathbf{n}=10\end{array}$ & $\begin{array}{c}\text { Prednisona } \\
\mathbf{n}=\mathbf{1 0}\end{array}$ & $\boldsymbol{p}^{*}$ \\
\hline Día 1 & $1,13 \pm 0,18$ & $0,24 \pm 0,09$ & 0,0001 \\
Día 3 & $1,34 \pm 0,16$ & $1,31 \pm 0,29$ & 0,650 \\
Día 5 & $1,56 \pm 0,15$ & $1,43 \pm 0,33$ & 0,449 \\
Día 7 & $1,76 \pm 0,14$ & $1,56 \pm 0,20$ & 0,046 \\
\hline
\end{tabular}

$* p<0,05$

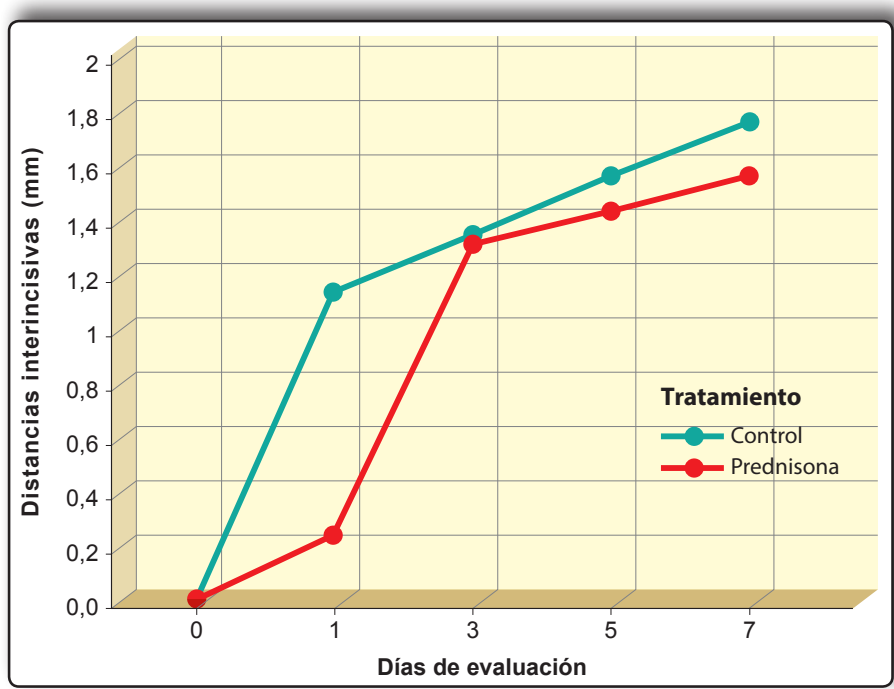

Figura 2. Distancia interincisiva $(\mathrm{mm})$ según tratamiento y por días de evaluación. 
y para fijarlo se empleó una resina fotopolimerizable (Transbond XT $3 \mathrm{M}$ ).

El disyuntor ortodóntico se mantuvo en los dientes de las ratas durante 7 días, procediéndose a hacer las mediciones de las distancias interincisivas en los días 1 , 3, 5 y 7, para facilitar la determinación de las variaciones en el movimiento con una medición directa (figura 1).

La administración de prednisona fue sólo al grupo experimental, a dosis de $20 \mathrm{mg} / \mathrm{kg}$ cada 12 horas por vía intraperitoneal, 5 días antes de la colocación del disyuntor ortodóntico, al momento de colocado el aparato y al primer, tercer y quinto día ${ }^{(3,4)}$.

\section{Análisis estadístico}

Los datos fueron agrupados, expresándose en valores medios \pm error estándar, con un intervalo de confianza del $95 \%$, teniendo una $p<0,05$ para considerar significativos los hallazgos. Se aplicó análisis de varianza y la prueba U de Mann-Whitney para establecer la significancia estadística entre el grupo control y el grupo experimental.

\section{RESULTADOS}

Las ratas que recibieron prednisona presentaron disminución del movimiento dental con respecto a las ratas que no recibieron, siendo más evidente en los días 1 y 7 con $p<0,0001$ y $p<0,046$; respectivamente (tabla 1 , figura 2), presentando, el grupo control 1,13 $\pm 0,18 \mathrm{~mm}$ y grupo con prednisona $0,24 \pm 0,09 \mathrm{~mm}$, de distancia interincisiva el $1^{\mathrm{er}}$ día de evaluación; mientras que el $7^{\mathrm{mo}}$ día, el grupo control tuvo una distancia interincisiva de 1,76 $\pm 0,14 \mathrm{~mm}$ y el grupo con prednisona de 1,56 $\pm 0,20$ mm (tabla 1) (U Mann de Whitney, $p<0,05$ ). A los días $3^{\text {ro }}$ y $5^{\text {to }}$ de evaluación, no se encontraron diferencias significativas entre los grupos $(p>0,05)$.

\section{DISCUSIÓN}

El movimiento dental está influenciado por muchos factores, entre los que se encuentran las prostaglandinas, quienes aceleran la resorción ósea incrementando el movimiento en cuestión ${ }^{(5)}$.

Los resultados obtenidos en el estudio indican que el movimiento dental disminuyó por efecto de la administración de prednisona (tabla 1; figura 1), esto se explica debido a que los corticoides interfieren con el acoplamiento de la resorción y el ciclo de reposición en el hueso normal, lo que se traduce en una reducción de la formación ósea y el aumento de la resorción ${ }^{(6)}$.

Los hallazgos concuerdan con los estudios realizados por Henriques et al (1), quienes determinaron que la dexametasona inhibe la reabsorción ósea y el movimiento dental durante el periodo inicial.

Lacerda et al (7), demostraron que los corticoides y otros inmunosupresores reprimen la acción de algunos linfocitos $\mathrm{T}$ que aceleran la destrucción fisiológica del hueso, y por lo tanto disminuyen el movimiento dental; otros estudios, realizados por Kalia et al (4), demuestran que los corticoides administrados a corto plazo suprimen la resorción ósea y el movimiento dental; Colin et al (8) determinaron que el uso de corticoides suprime la actividad osteoclástica afectando la reabsorción ósea y por ende en el movimiento dental; Ong et al ${ }^{(8)}$, encontraron que la prednisolona disminuye la resorción y aposición óseas, logrando que el movimiento dental se retrase, al modificar la expresión de algunas moléculas como el receptor de la hormona de crecimiento (GHR) y el receptor del factor de crecimiento insulínico tipo I (IGF-IR) de los tejidos dentales.

Sin embargo otros estudios, en contraste a lo obtenido, encontraron que el uso de corticoides acelera el movimiento dental y la reabsorción ósea. Así, Mohammed (9) determinó que el uso de metilprednisolona a corto plazo acelera el movimiento dental en ratas, mientras que a largo plazo lo retrasa. Bartzela et al (2), señalan que los glucocorticoides y los eicosanoides aceleran el movimiento dental; Verna et $a l^{(10)}$, refiere que el uso agudo de corticoides mejoran la reabsorción ósea y por lo tanto incrementan el movimiento dental. Gonzales et al (11), demostraron que los glucocorticoides, como la prednisolona, no modifican el movimiento dental en ratas.

\section{CONCLUSIÓN}

La prednisona, administrada porvía intraperitoneal, a dosis de $20 \mathrm{mg} / \mathrm{kg}$, reduce la longitud del movimiento dental en ratas en el $1^{\mathrm{er}}$ y $7^{\mathrm{mo}}$ día de tratamiento.

\section{REFERENCIAS BIBLIOGRÁFICAS}

1. Henriques LA, Lima R, Borges L, Trindade AM, Tanaka O. The action of corticosteroids on orthodontic tooth movement: a literature review. Dental Press Journal of Orthodontics 2012; 17(6): 20e1-e5.

2. Bartzela T, Türp J, Motschall E, Maltha J. Medication effects on the rate of orthodontic tooth movement: a systematic literature review. American Journal of Orthodontics and Dentofacial Orthopedics 2009; 135(1): 16-26.

3. Retamoso L, Knop L, Shintcovsk R, Maciel J, Machado A, Tanaka O. Influence of anti-inflammatory administration in collagen maturation process during orthodontic tooth 
movement. Microscopy Research and Technique 2011; 74(8): 709-13.

4. Kalia S, Melsen B, Verna C. Tissue reaction to orthodontic tooth movement in acute and chronic corticosteroid treatment. Orthod Craniofacial Res 2004; 7(1): 26-34.

5. Knop LA, Shintcovsk RL, Retamoso LB, Ribeiro JS, Tanaka OM. Non-steroidal and steroidal anti-inflammatory use in the context of orthodontic movement. European Journal of Orthodontics 2012; 34(5): 531-5.

6. Ong CK, Walsh LJ, Harbrow D, Taverne AA, Symons AL. Orthodontic tooth movement in the prednisolonetreated rat. Angle Orthodontist 2000; 70(2): 118-25.

7. Lacerda R, Mesquita $\mathrm{M}$, Torres $\mathrm{R}$, Martins $\mathrm{M}$, Gomes M. Immunosuppressants: implications in orthodontics. Dental Press Journal of Orthodontics. 2012; 17(2): 55-61.

8. Ong CK, Joseph BK, Waters MJ, Symons AL. Growth hormone receptor and IGF-I receptor immunoreactivity during orthodontic tooth movement in the prednisolone-treated rat. Angle Orthodontist 2001; 71(6): 486-93.

9. Mohammed HS. Orthodontic tooth movement in low- dose for different courses methylprednisolone-treated rats. International Journal of Advanced Biological Research 2012; 2(3): 545-51.

10. Verna C, Hartig L, Kalia S, Melsen B. Influence of steroid drugs on orthodontically induced root resorption. Orthodontic Craniofacial Research 2006; 9(1): 57-62.

11. Gonzales C, Hotokezakab H, Matsuoc K, ShibazakidT, Yozgatiana J, Darendelilere A, et al. Effects of Steroidal and Nonsteroidal Drugs on Tooth Movement and Root Resorption in the Rat Molar. Angle Orthodontic 2009; $79(4): 715-26$.

Manuscrito recibido el: 07/o8/14

Aceptado para su publicación el: 04/o9/2014

\section{Correspondencia:}

Nombre: $\quad$ Eliberto Ruiz Ramírez

Dirección: Calle José Gálvez 296, Urb. Condevilla

e-mail:

eruizramirez86@gmail.com 